\title{
Statistical UDWDM-PONs operating with ONU lasers under limited tunability
}

\author{
Vicent Sales, Josep Segarra, Member, IEEE, Victor Polo, and Josep Prat, Member, IEEE
}

\begin{abstract}
We explore a new wavelength management for ultra-dense WDM-PONs with randomly distributed ONU wavelengths, controlled with limited thermal tunability. Static and dynamic wavelength assignment paradigms and heuristic assignment schemes for spectrum management are proposed, simulated and compared. ONU acceptances in activation processes and ONU availability ratios up to $99.99 \%$ are obtained in standard environmental conditions.
\end{abstract}

Index Terms-Limited thermal tunability, Optical Network Unit (ONU), Statistical Ultra Dense Wavelength Division Multiplexing, Passive Optical Network, wavelength assignment.

\section{INTRODUCTION}

$\mathrm{N}$ OWADAYS PONs based on TDM are being installed following GPON and EPON standards, which can be upgraded increasing the bit rate at the OLT and ONU transceivers to XG-PON [1] and 10G EPON [2] by overlaying additional wavelengths at higher bit rates to attain growing user bandwidth demands; they compatibly keep the Optical Distribution Network (ODN) based on splitters and the TDM basis running up to $10 \mathrm{~Gb} / \mathrm{s}$, with higher speed requirements and increased power consumption. To further increase the network capacity, several proposals have been introduced, including WDM [3], hybrid WDM/TDM [4], TWDM [5] and OFDM PONs [6], although, the low cost and backwards compatibility requirements are not always yet fulfilled.

WDM-PONs can offer dedicated wavelengths to residential customers as well to business and mobile base stations at any non-shared bit rate, therefore increasing the network capacity greatly. By using new coherent receivers and spacing the channels at few $\mathrm{GHz}$, Ultra Dense WDM (UDWDM) can implement the "wavelength-to-the-user" concept over an splitter based ODN. Coherent systems provide excellent wavelength selectivity, high sensitivity and therefore long reach extension [7], [8], thus leading to the UDWDM-PON. Low cost coherent terminals can be potentially suitable for deployment in access networks with typical consumer electronics [3], [9], delivering e.g. $1 \mathrm{~Gb} / \mathrm{s}$ to a single residential user. This realization requires new control

Manuscript received September 17, 2014. This work was supported in part by the European COCONUT Project (G.A. 318515) and the Spanish Ministry of Science and Innovation under grant TEC2011-25215 (ROMULA).

The authors are with the Universitat Politècnica de Catalunya (UPC), Teoria del Senyal i Comunicacions, Barcelona (TSC), E-08034, Spain (e-mail: vicent@tsc.upc.edu). mechanisms of UDWDM to organize the ultra-dense spectrum distribution.

When implementing coherent reception, a tunable laser (TL) is used as local oscillator and another TL can be employed for transmission. Typically, in core networks, fully TL are used, but in access they can be expensive or complex to control. In contrast, low cost TLs with narrow tunability like conventional DFB-lasers, with heating/cooling control, present very simple and reliable tuning function. Its operating temperature changes the wavelength in about $0.1 \mathrm{~nm} /{ }^{\circ} \mathrm{C}[10]$, [11]; providing a continuous but limited tuning range. On the other hand, typical production of DFB-lasers yields random emitting wavelengths varying over few nanometers range [10]; this range can be extended to the intended PON band, of e.g. 10 to $40 \mathrm{~nm}$, by some practical means for further study, where users could acquire non-preselected ONUs.

To overcome these practical limitations in FTTH, where all ONUs should be equal or non-preselected for simple provisioning, statistical UDWDM multiplexing was proposed in [12]. This principle sets that the wavelengths in the ONUs have a random distribution in a band, and that they are thermally tuned with limited range to different ultra-dense wavelength channels avoiding collisions.

When a new ONU is introduced at the PON, a wavelength is assigned to it within its limited thermal tuning range; however, it could interfere with existing connections; therefore, there is a collision contention probability to be avoided by controlling the operating wavelength of the lasers. Thermal tuning has also been proposed in Time and Wavelength-Set Division Multiplexing (TWSDM) for TWDM [5] and in Dynamic Spectrum Management (DSM) [10], [13], using a cyclic AWG at the OLT with a limited number of passband channels.

In this work, static and dynamic wavelength assignment algorithms for spectrum management of UDWDM-PON are proposed and dimensioned for the first time, in activation process and operation. To validate key practical assumptions, before in section II, experimental DFB-laser characterization is reported, showing a proper transmission behavior at the considered temperature ranges.

\section{EXPERIMENTAL DFB-LASER CHARACTERIZATION}

\section{A. Limited thermal tunability}

The collision contention probability will directly depend on the thermal tuning range of the laser, limited by the 
operational temperature range and the heating/cooling consumption.

Operating temperatures up to $90^{\circ} \mathrm{C}$ have been reported using DBR lasers [14], but we will limit the temperature range up to $55^{\circ} \mathrm{C}$ by employing DFB-lasers, hence reducing the power consumption and maintaining directly DPSK modulated laser performances [9]. The complete temperaturedependent device behavior is a combination of shifts of the refractive index as well as the grating reflection wavelength in the laser cavity [15]. The temperature range also depends on the ambient temperature of the ONU environment.

Fig. 1 illustrates the measured wavelength tuning variation of a DFB-laser as a function of the temperature control, exhibiting the typical very constant linear variation of 0.08 $\mathrm{nm} /{ }^{\circ} \mathrm{C}$ approximately between 18 to $50^{\circ} \mathrm{C}$, for bias currents of 60,80 and $100 \mathrm{~mA}$; it spans for $2.5 \mathrm{~nm}$ approx., thus enabling the DFB-laser to tune up to 50 channels spaced at $6.25 \mathrm{GHz}$ [16]. This temperature variation range is easily reached with a Peltier, and also even just by heating applying an offset, depending on the ambient temperature range.

When a new ONU joins or resumes operation in a PON, an activation procedure at the OLT has to identify and accept the entrant user, and a new wavelength is allocated to the ONU, close to its nominal wavelength and within its limited thermal tuning range. If the neighboring wavelengths channels are already occupied by other ONUs in service, the new ONU has to "jump" those wavelengths. This jumping action can be done at low power in order to reduce the interference, or/and executed very fast to minimize the interfering time of the working wavelengths. Simple and fast tuning methods for temperature tunable DFB-lasers have been presented, reducing the interfering time in the jumped channel from 20 seconds to less than $1 \mu \mathrm{s}$ [17]. The impact of this short interruption, in modern services is for further study. An activation procedure has been presented in [18].

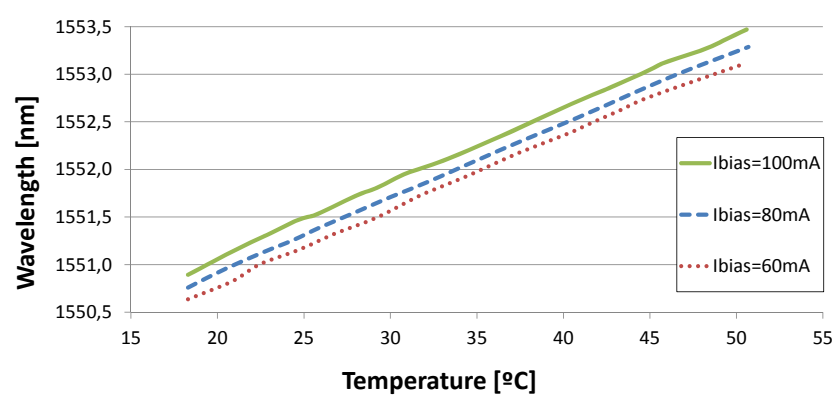

Fig. 1. DFB-laser wavelength vs. temperature for different $\mathrm{I}_{\text {bias }}$ currents.

\section{B. Transmission performance}

Thermally tuned directly phase modulated DFB-lasers at the ONU transmitting in DPSK coherent UDWDM at 1.25 $\mathrm{Gb} / \mathrm{s}$ [9] are measured in terms of received optical power and Bit Error Rate (BER) at OLT reception against temperature variations of the ONU transmission laser. The reception at the OLT is realized with a power stabilized laser tuned at the same wavelength. It is shown for a standard $4 \mathrm{MHz}$ linewidth $1 \mathrm{~mW}$ DFB-laser in Fig. 2, together with the received power, at laser bias currents of $60 \mathrm{~mA}$ and $100 \mathrm{~mA}$. The received power remains with a variation of $3 \mathrm{~dB}$ approx., while the BER detection with a coherent homodyne receiver is kept below the FEC margin of $10^{-3}$ up to $60^{\circ} \mathrm{C}$. Above it, the performance is degraded, because of the power reduction, the phase modulation bandwidth reduction and the linewidth increase.

These results validate the parameters that will be used in the following analysis scenarios.

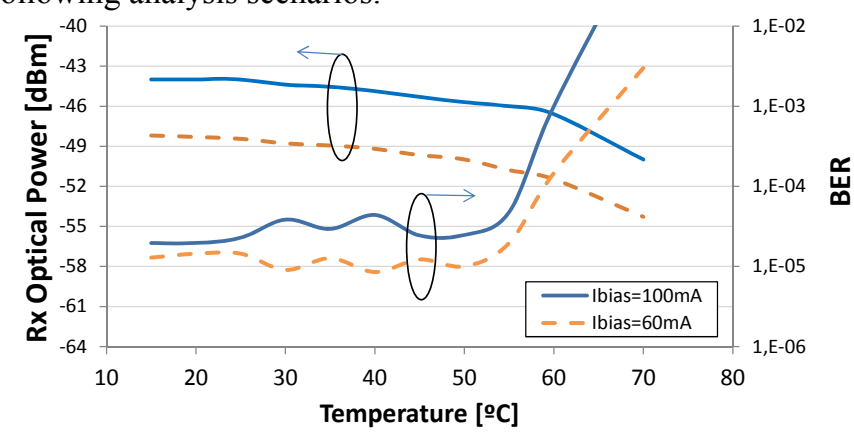

Fig. 2. DFB-laser received power and BER against temperature at OLT.

\section{Statistical UDWDM WAVELENGTH AssignMENT}

\section{A. The assignment paradigms}

We address the ONU wavelength management problem by proposing heuristic algorithms controlled by the OLT. But primarily, in order to allocate each ONU random wavelength with limited tunability to a specific managed assigned wavelength, avoiding collision contentions, two assignment paradigms are considered [19]:

- Static Wavelength Assignment (SWA), where once wavelengths are assigned, these stay static.

- Dynamic Wavelength Assignment (DWA), where wavelengths reassignments are made, also to satisfy network changes in harsh environmental temperature conditions.

\section{B. The heuristic assignment schemes}

The ONUs own a Narrowly Tunable Laser (NTL) whose wavelength has a random value distributed in uniform probability over a specific optical band for down/up transmission in the same wavelength (and another NTL if down/up is done in different wavelengths). The optical band is divided in a number of equally and narrowly spaced channels. When an ONU is activated, a free channel is assigned to its NTL in the window defined by its limited tuning range; if there is no channel availability in the window, the ONU is rejected. The signal bandwidth is lower than the channel spacing; hence, the separation between two lasers can be lower than the channel spacing, because the laser wavelength does not need to be in the center of the channel. The proposed heuristic channel assignment schemes in this scenario are:

- First Fit (FF), selects the free channel closer to the random ONU wavelength [12].

- Maximum Scattering (MS), selects the free channel providing the maximum distance between two busy channels. 
- Maximum Admittance (MA), assigns the free channel giving the maximum options of admission when a future new request arrives at the same assigned channel.

An OLT internal architecture based on splitters to combine the UDWDM lights covers the number of deployed ONUs and the whole working optical band, with an optical amplifier if necessary to satisfy the power budget.

In order to evaluate the assignment schemes, simulations are realized by applying the Monte-Carlo method to find the number of channels needed in a PON, by calculating the rejection probability due to inevitable channel collision. Each ONU has two NTLs at two different optical bands for down and up-transmission, respectively, and an ONU is rejected when one or both NTLs cannot be allocated.

The channel spacing is taken to be $6.25 \mathrm{GHz}$ as using coherent detection [16], which can be allocated at the C-band between 192.1 and 196.1 THz (32 nm aprox.). The minimum wavelength separation between two adjacent channels is 3 $\mathrm{GHz}$ [16]. We have evaluated that the interference from nonadjacent channels with a typical Side Mode Suppression Ration (SMSR) higher than $35 \mathrm{~dB}$ has a penalty of less than 1 $\mathrm{dB}$, and therefore is not affecting. The tunability is assumed to be $0.1 \mathrm{~nm} /{ }^{\circ} \mathrm{C}$, with a range of $+/-10^{\circ} \mathrm{C}$, which can be achieved with a Peltier or just by heating the original wavelength $+10^{\circ} \mathrm{C}$ and then incrementing or decrementing it up to $10^{\circ} \mathrm{C}$.

Fig. 3 illustrates an example of the spectrum distribution in an activation procedure with only 20 ONUs for the sake of clarity, showing the random wavelengths distribution and the final tuned wavelengths achieved with the three proposed heuristic schemes.

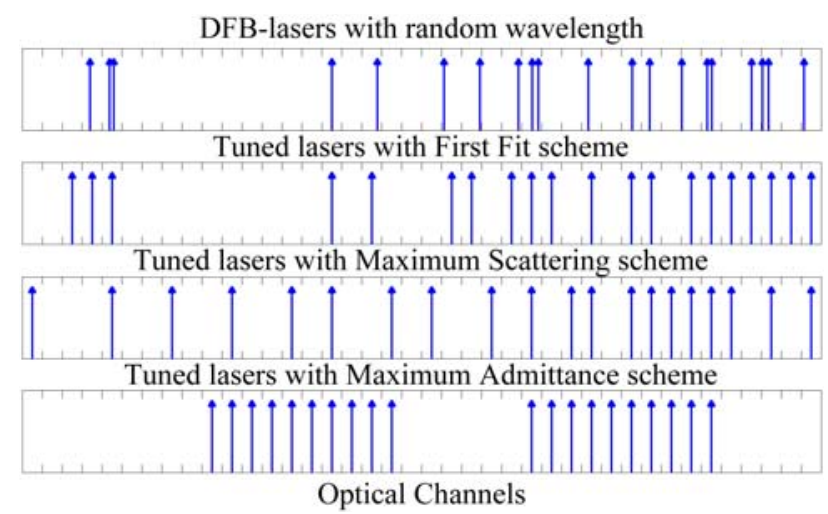

Fig. 3. Spectrum distribution before (upper) and after assignment for FF, MS and MA heuristic assignment schemes, respectively.

As we can observe, FF assignment scheme allocates the wavelengths neighboring the original random wavelengths, selecting the free channels closer to the original wavelengths; it requires minimum tuning stress and consumption [12]. MS scheme situates the wavelengths in a more spaced manner, providing free channels more uniformly. Finally, MA scheme keeps free the channels with more probability to be requested in the internal band and at the band limits, where a part of its tuning window is out of band and cannot be used; these are the central and side channel regions, respectively.
In the MA scheme the resulting blocks of assigned channels are smaller than the NTL window, and therefore when a new request arrives over any formed block it can be allocated.

The deployed number of ONUs is taken to be 256. In the activation process, when using SWA, the rejection probability is evaluated as a function of the number of required channel slots. FF is the simplest assignment scheme and is used as a basis for comparison. As shown in Fig. 4, MS exceeds FF, but MA provides the best results. When using MA, the rejection probability of $10^{-3}(0.1 \%$ rejection or the highest $99.9 \%$ acceptance) is achieved with only 305 channels. With the same acceptance ratio, when employing MS, 335 channels are needed; and while using FF, 360 channels are employed.

Now we consider the DWA case: existing wavelengths can be reassigned in the activation process to attain a new entering laser that finds all channels busy in its tunable window. In this window a connected user is searched, beginning in the lowest frequency, with at least a free channel in its particular window; if a free channel is found, the working wavelength is reassigned by applying any of the heuristic schemes, and the liberated channel is assigned to the incoming ONU. DWA is more complex to implement, but simulations with the same former channel tunability exhibit an improvement of the rejection probability.

Again, for 256 active ONUs to be allocated, the rejection probability against the number of deployed channels is illustrated with DWA in Fig. 4. The DWA forces FF to scatter the NTLs more uniformly in the band, as MS does, and therefore, the results are very similar. DWA combined with MA furnishes the best results, showing a rejection of $0.1 \%$ with only 290 channels, improving the SWA paradigm.

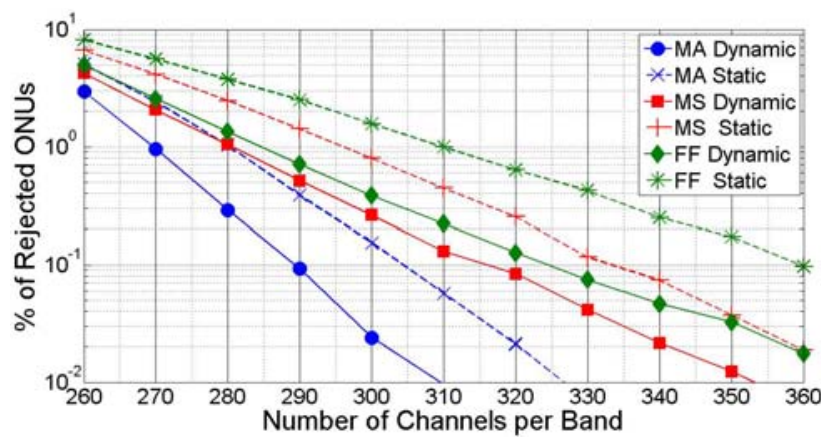

Fig. 4. Activation rejection probability vs. number of channel slots using SWA or DWA paradigms and FF, MS and MA heuristic assignment schemes, successfully allocating 256 ONUs.

\section{Wavelength reassignment during operation}

Another scenario where to apply DWA is when environmental conditions change. Commercial temperature range corresponding to indoor equipment defines a moderate variation, but industrial range matching outdoor equipment demands great temperature changes, as night/day and seasonal transitions may exhibit. Therefore, when a temperature variation occurs, the NTL changes its tunable window and, when a large variation reaches a value where the NTL cannot keep the assigned working channel, a reassignment is needed. 
Hereafter, a continuous monitoring of the laser window thermal tunability at the ONU is needed to track the assigned channel and a reassignment is requested by the OLT to be made when the present assigned channel cannot be sustained. At the OLT an optical spectrum monitoring is continuously tracking all wavelengths present at the PON, controlling that they are correct in power and allocation; not only for activation purposes but also making decisions about existing channel reallocations. It employs an OLT-ONU negotiation based on operation and maintenance (O\&M) messages. The reallocation decisions of the ONU wavelength to a new channel of its current tunable window can be executed with any of the former heuristic schemes.

To show this external temperature variation scenario, we set the case where $50 \%$ of the ONUs are outdoors and $50 \%$ of the ONUs are indoors, suffering great and low deviations, respectively. Smooth daily temperature changes of $20^{\circ} \mathrm{C}$ are applied to outdoor ONUs and $5^{\circ} \mathrm{C}$ to indoor ONUs. Again, simulations with the same former conditions are performed; during operation, the ONUs which cannot hold its current channel are reassigned, applying any of the heuristic schemes. In order to evaluate the performance of the system, the ONU Availability Ratio (OAR) is defined as the ratio of available time to total operating time of all allocated channels, according to the performance parameters and objectives outlined for transmission systems and media [20]. OAR is found and, in Fig. 5, the contention ratio (1-OAR)\% illustrates that the network adapts dynamically to temperature changes. Now in operation, the performance of the algorithms has changed. FF exceeds MS because in FF the NTLs are tuned close to its random central wavelengths and they can compensate large temperature changes keeping the proper assigned channel by using only the temperature control. MA suffers the same effect, but MA reallocates much better the channels than FF and MS and, hence, MA achieves the best results. With MA an OAR of $99.99 \%$ with 540 channel is obtained.

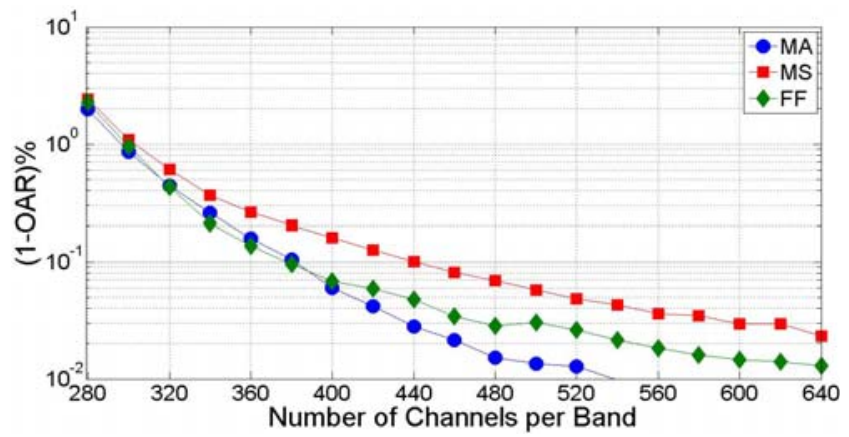

Fig. 5. ONU availability ratio when using DWA and FF, MS and MA heuristic assignment schemes to adapt to environmental temperature changes.

\section{CONCLUSIONS}

An efficient statistical UDWDM management paradigm has been proposed and dimensioned for new PONs, where wavelengths in the ONUs have a non-preselected random distribution in a band, and they are thermally tuned to different channels avoiding collisions.

An experimental DFB-laser characterization exhibits a good transmission performance in a realistic temperature range, validating the parameters used in the simulation scenarios.

To manage the wavelengths efficiently, we proposed Static and Dynamic Wavelength Assignment paradigms combined with three heuristic assignment schemes: First Fit, Maximum Scattering and Maximum Admittance. Simulations results show that Dynamic Wavelength Assignment paradigm associated with Maximum Admittance scheme provides the best results to avoid channel collisions and to adapt to environmental changes, in terms of ONU acceptances in the activation process up to $99.9 \%$, and ONU availability ratios, up to $99.99 \%$.

\section{REFERENCES}

[1] ITU-T G.987, "10-Gigabit-capable passive optical network (XG-PON) systems", Geneva, Switzerland, 2012.

[2] 10G EPON standard IEEE P802.av, www.ieee802.org/3/

[3] Y. C. Chung, "Recent advancement in WDM-PON technology," in Proc. ECOC, Th.11.C.4, Geneva, Switzerland, 2011.

[4] J.Prat, et al., "Demonstration and field trial of a scalable and resilient hybrid ngPON," in Proc. ECOC, Tu.6.C.3, Geneva, Switzerland, 2011.

[5] D. van Venn et al., "System demonstration of a time and wavelength-set division multiplexing PON," in Proc. ECOC, We.3.F.2, London, UK, 2013.

[6] D. Qian et al., "A novel OFDM-PON architecture with source-free ONUs for next-generation optical access networks," IEEE Photonics Technology Letters, vol. 21, no. 17, pp. 1265-1267, 2009.

[7] H. Rohde et al., "Next generation optical access: 1 Gbit/s for everyone," in Proc. ECOC, 10.5.5, Vienna, Austria, 2009.

[8] J. M. Fabrega et al., "Ultra-Dense, transparent and resilient ring-tree access network using coupler-based remote nodes and homodyne transceivers," in Proc. ICTON, Th.B3.3, Ponta Delgada, Azores, Portugal, 2009.

[9] I. N. Cano, et al., "Direct phase modulation DFBs for cost-effective ONU transmitter in udWDM PONs," IEEE Photonics Technology Letters, vol. 26, no. 10, pp. 973-975, 2014.

[10] N. Cheng et al., "Dynamic spectrum managed passive optical networks," IEEE Communications Magazine, vol. 49, no.11, pp. 86-93, 2011.

[11] T. Garrod et al., "High-power and high-efficiency distributed feedback (DFB) lasers operating in the 1.4-1.6 $\mu \mathrm{m}$ range for eye-safe applications," in Proc. SPIE 8605, High-Power Diode Laser Technology and Applications XI, 86050I, S. Francisco, CA, USA, 2013.

[12] I. Cano et al., "Dimensioning of OFDMA PON with non-preselectedindependent ONUs sources and wavelength-control," in Proc. ECOC, Tu.5.C.2, Geneva, Switzerland, 2011.

[13] D. Piehler, "PICs in PONs", in Proc. OFC, NTu1J.6, Los Angeles, CA, USA, 2012.

[14] S. Pachnicke et al., "First demonstration of a full C-band tunable WDMPON system with novel high-temperature DS-DBR lasers," in Proc. OFC, W.3.G.2, S. Francisco, CA, USA, 2014.

[15] H. Zappe, "Laser diode microsystems (Microtechnology and MEMS)," Springer, 2003.

[16] J. Prat et al., "Simple intradyne PSK system for udWDM-PON", in Proc. ECOC, We.2.B.2, Amsterdam, The Netherlands, 2012.

[17] V. Polo et al., "DFB laser reallocation by thermal wavelength control for statistical udWDM in PONs," in Proc. ECOC, P.4.13, Cannes, France, 2014.

[18] C. N. Ververidis et al., "Control and management requirements for a coherent ultra-dense WDM PON for lambda to the user access networks," in Proc. ICTON, Mo.C3.6, Graz, Austria, 2014.

[19] M. P. McGarry et al., "Bandwidth management for WDM EPONs," IEEE Journal of Optical Networking, vol. 5, n.9, pp. 637-654, 2006.

[20] ITU-T G.827, "Availability performance parameters and objectives for end-to-end international constant bit-rate digital paths," Geneva, Switzerland, 2003. 